\title{
Viscous magnetization of loess/palaeosol samples from Bulgaria
}

\author{
Diana Jordanova, Gergana Yancheva, and Valentin Gigov \\ Geophysical Institute, Bulg. Acad. Sci., Acad. G. Bonchev Str., bl.3, 1113 Sofia, Bulgaria \\ (Received June 6, 2000; Revised October 6, 2000; Accepted December 1, 2000)
}

\begin{abstract}
The processes of acquisition of viscous remanence and its decay in zero magnetic field are studied for three sets of palaeosol and loess samples. A linear behaviour of VRM vs. $\log t$ minus is obtained for $10-10^{4}$ min. to 30 days of acquisition experiments. Linear correlation between the obtained coefficients of viscous acquisition normalized to saturation magnetization $(\mathrm{Sa} / \mathrm{Js})$ or frequency dependent magnetic susceptibility $(\mathrm{Sa} / \chi \mathrm{fd} \%)$ and the ratio of susceptibility to saturation magnetization $(\chi / \mathrm{Js})$, suggests that ultra fine SP/SD grains are mainly involved in the process of viscous acquisition. Non-linear VRM vs. $\log t$ behaviour is observed during viscous decay in $\mu$-metal shielded space, being most pronounced for the first $10 \mathrm{~min}$. of the experiment. It is supposed that this phenomena is due to the presence of composite grains (core/shell structure), formed as a result of low-temperature oxidation. The latter increases towards older palaeosol units.
\end{abstract}

\section{Introduction}

Viscous remanent magnetization (VRM) is the most common secondary remanence, acquired by rocks during their exposure to the Earth's magnetic field. VRM plays an important role in loess/soil sediments, where a significant part of the total remanence is of viscous origin (Hus and Geeraerts, 1986; Heller and Evans, 1995). Having in mind the increased hardness of the viscous remanence with increasing acquisition time, found for natural samples (Prevot, 1981), the question about successful separation of the characteristic remanence is very important. The main mechanism of viscous acquisition is considered to be thermal agitation of viscous moments (Dunlop and Ozdemir, 1997). The main contribution to the viscosity comes from the fraction of unstable grains with sizes just at the critical superparamagnetic/single domain (SP/SD) threshold (Dunlop, 1983; O’Reilly, 1984). Possible contribution from a multidomain (MD) fraction would be masked to a great extent because the viscosity of MD particles is significantly lower than that of SP/SD grains. In the case of the prevailing role of the fine-grained fraction, which is the situation for palaeosols (Fine et al., 1995; Hunt et al., 1995; Heller and Evans, 1995), such MD viscous effects could be identified only in the unweathered loess samples, containing mostly detrital MD grains.

The present study is aimed at identifying the cause of peculiarities in acquisition and decay of VRM in palaeosol and loess sediments from a core in NE Bulgaria (near the village of Koriten). It consists of seven loess beds (L1-L7) and six interbedded palaeosols (S1-S6) with a total thickness of $34 \mathrm{~m}$. Loose material was gathered at $10 \mathrm{~cm}$ intervals.

Rock magnetic characteristics obtained through hysteresis measurements are discussed in an earlier paper (Jordanova and Petersen, 1999). The main results point to the prevailing

Copy right(C) The Society of Geomagnetism and Earth, Planetary and Space Sciences (SGEPSS); The Seismological Society of Japan; The Volcanological Society of Japan; The Geodetic Society of Japan; The Japanese Society for Planetary Sciences. role of the pedogenic magnetite/maghemite fraction in determining the magnetic behaviour of palaeosol samples. This fraction leads to a significant magnetic enhancement and high values of percent frequency dependent magnetic susceptibility (up to $12-14 \%$ ) and lower coercivity parameters (Hc, Hcr, Hcr') in the older palaeosol horizons.

The present set of experiments is aimed to shed more light on the behaviour of the pedogenic fraction in the grain size range, which determines the viscosity effects. Having in mind the obtained strong size-dependence of the viscosity coefficients ( $d^{-n}$ with $n=1-1.5$; Dunlop, 1981) differences in VRM characteristics can give additional information about the grain-size distributions in different palaeosol units.

\section{Samples and Methods}

Remanences were measured with a spinner magnetometer JR-4 (AGICO, Brno), AF demagnetizations were carried out with a MOLSPIN device with a tumbling mechanism. Triple $\mu$-metal boxes were used for zero-field storage (ZFS) tests, with residual magnetic field inside up to $5 \mathrm{nT}$. Kappa-bridge KLY-2 was used for susceptibility measurements.

Samples were prepared and studied in three sets. The first set is a collection of 45 non-oriented cubes $(2 \times 2 \times 2 \mathrm{~cm})$ which were cut from solid pieces of material. Thus, the "in situ" position of the material could not be obtained, but we use an arbitrary sample coordinate system, chosen in a way, so that the maximum of the measured orthogonal components of the remanent signal is assigned to a $Z$-direction. The following procedures are applied subsequently: 1) initial NRM measurements and definition of the arbitrary coordinate system of each sample; 2 ) zero-field storage (ZFS) in $\mu$-metal space for 30 days, 3 ) immediately after the measurements of the remaining signal, samples were placed in the laboratory geomagnetic field with their $x$-axis parallel to the local north direction and the $z$-axis pointing downward. After 30 days of exposure, the remanence $\left(\mathrm{NRM}_{+z}\right)$ was measured and 4) 
$I^{\text {st }}$ position
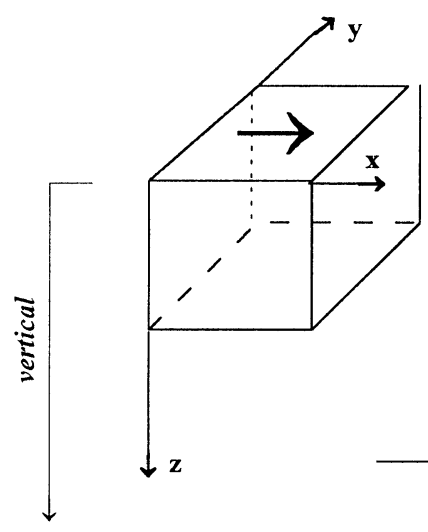

II ${ }^{\text {nd }}$ position

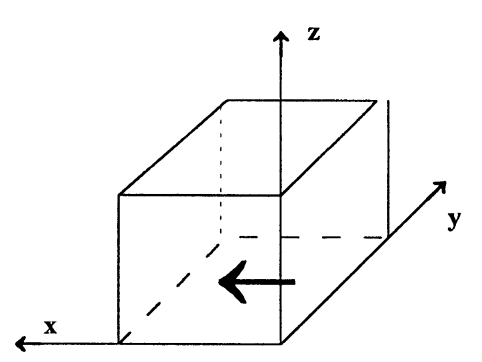

local North direction

Fig. 1. Schematic presentation of the viscous cleaning experiment in laboratory magnetic field using two exposure positions of the samples in respect to the field.

samples were rotated at $180^{\circ}$ around their $y$-axis in order to destroy the already acquired VRM during the first exposure time (Fig. 1). After 30 days, the remanence was measured again $\left(\mathrm{NRM}_{-z}\right) 5$ ) After subsequent storage of 1 month in a position parallel to the direction of laboratory field, samples were step-wise AF demagnetized up to $20 \mathrm{mT}$ peak field in steps of $2.5 \mathrm{mT}$ and coercivity spectra were obtained.

The second set of samples consists of 12 non-oriented cubes, cut and marked in the same way as the first set. All the samples from this set were taken from palaeosol units. The intention was to follow the process of viscous acquisition with time for samples with strong pedogenic enhancement and to check for the presence of any peculiarities apart from a linear VRM $(\log t)$ behaviour. An initial ZFS of 5 to 6 days, applied in order to obtain similar initial state as for the set 1 , preceded the process of VRM acquisition. The latter was monitored in regularly spaced $\log (t)$ intervals. Immediately after the last measurement, samples were inverted and the process of re-acquisition of viscous remanence (e.g. destruction of the previous VRM and acquisition of a new VRM in the opposite direction) was also monitored (see also Fig. 1). A few samples were then used to repeat again the VRM acquisition along the $(+z)$ direction after the preceding one along the $(-z)$ direction.

The third set of samples was prepared in a different way. It consists of 20 cubes both from loesses and palaeosols. After mechanical grinding of the material, it was passed through a $0.2 \mathrm{~mm}$ sieve and artificial samples were prepared mixing the mechanical fractions with water and a small amount of Plaster of Paris. This procedure was undertaken in order to assure that samples are free of remanence components, which are initially present in any rocks. After drying of the samples, they were AF demagnetized at $100 \mathrm{mT}$ and left in the laboratory field. VRM acquisition process was monitored through regular measurements of the remanent magnetization during a period of 30 days. The viscosity acquisition coefficients Sa were calculated from the slope of the $\log (t)$ vs. VRM time dependence. One sample from each palaeosol unit was used to study spontaneous decay of VRM in zero field. For this purpose, the sample was inserted into the holder of the JR-4 spinner magnetometer and continuous decay of VRM with time (up to 4 hours) was monitored. In order to check the role of different acquisition times on the coercivity of VRM, a few samples were subjected to a set of VRM acquisitions for 20 min., 1-2 days and 3 to 5 months. Subsequent step-wise AF demagnetization after each acquisition period, was carried out.

A complimentary sample (MGT), containing natural $\mathrm{Fe}_{3} \mathrm{O}_{4}$ crystals of $0.2 \mu \mathrm{m}$ size, was prepared so as to contain about $1 \%$ magnetite and subjected to viscous acquisition and subsequent decay of VRM in order to compare the behaviour of a sample of known magnetic grain size with the studied palaeosol samples.

\section{Experimental Results}

Viscous and stable remanence components, calculated for samples from set 1 after the viscous decay in zero field, and subsequent double exposure for VRM acquisition parallel $\left(\mathrm{VRM}_{+}\right)$and antiparallel $\left(\mathrm{VRM}_{-}\right)$to the laboratory magnetic field, are shown in Fig. 2. Comparing the values of the viscous and stable remanences, obtained by the two experimental procedures, the stratigraphic variation in the amounts of unstable (soft) magnetizations $\left(\mathrm{VRM}_{\mathrm{ZFS}}\right.$ and $\left.\mathrm{VRM}_{+-}\right)$ that have been subtracted, is obvious. The magnitude of $\mathrm{VRM}_{\text {ZFS }}$ is obtained as a vector subtraction of the stable remanence, $\mathrm{NRM}_{\mathrm{ZFS}}$, measured after 30 days of ZFS, from the initial NRM signal. $\mathrm{VRM}_{+-}$, defined as the viscous component following directional changes in the ambient field, has been calculated as: $\mathrm{VRM}_{+-}=1 / 2\left(\mathrm{NRM}_{+z}-\mathrm{NRM}_{-z}\right)$. The similarity in the values of stable remanence unaffected by viscosity treatments $\left[\mathrm{NRM}_{\mathrm{ZFS}}\right.$ and $\mathrm{NRM}_{+-}=1 / 2\left(\mathrm{NRM}_{+z}+\right.$ $\mathrm{NRM}_{-z}$ )] suggests that one and the same fraction of ferromagnetic grains carries the stable remanent signal. Consequently, the differences in the two viscous remanences $\left(\mathrm{VRM}_{\mathrm{ZFS}}\right.$ and $\left.\mathrm{VRM}_{+-}\right)$are brought about by other factor than efficiency of decay in zero field and viscous acquisition. Supposition that the presence of an external magnetic field (Earth's m.f.) would lower the energy barriers and enhance 


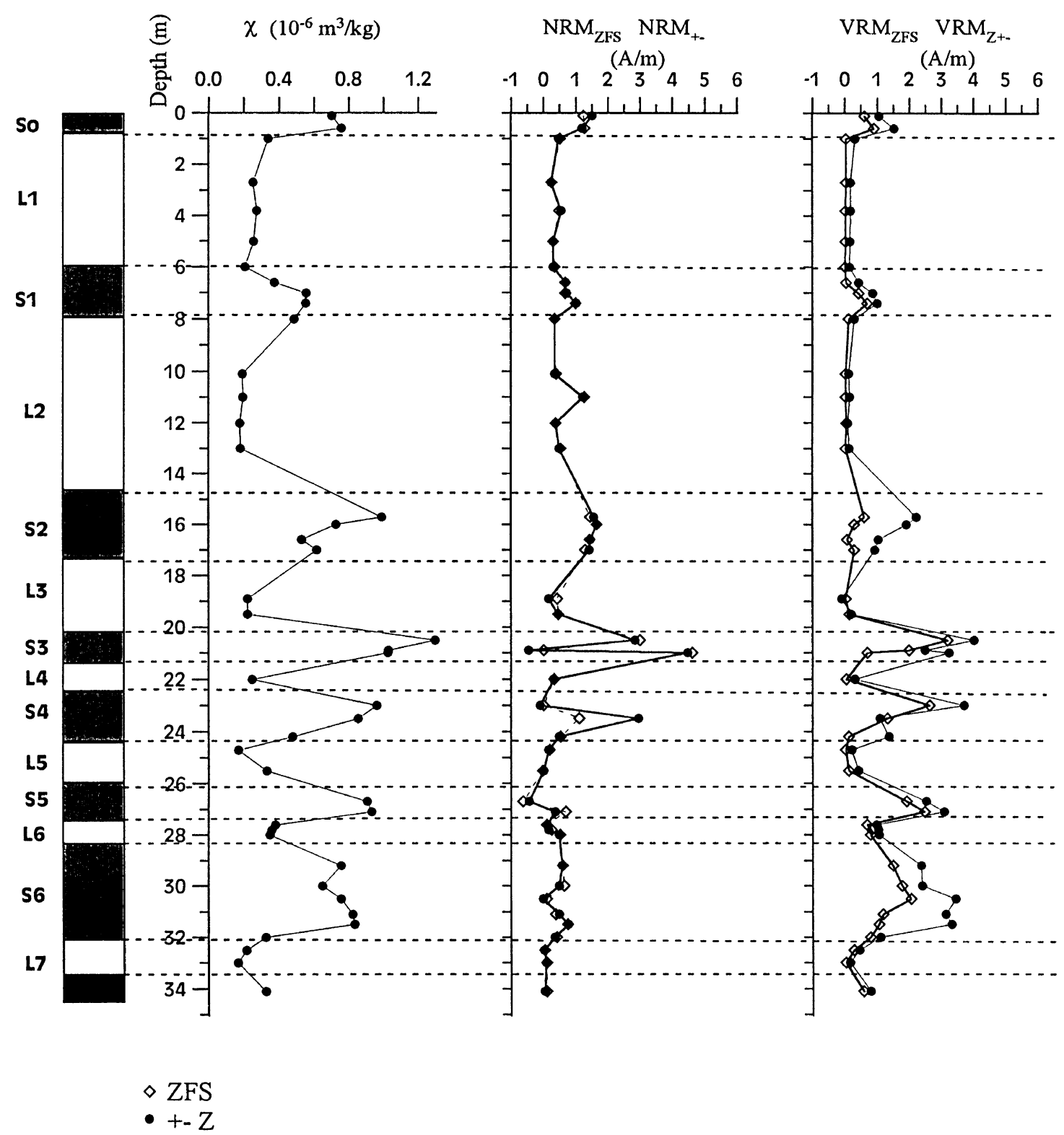

Fig. 2. Variations of susceptibility $(\chi)$, undecayed remanence signals after: i) 30 days of ZFS and ii) subsequent double exposure to the laboratory magnetic field $\left(\mathrm{NRM}_{\mathrm{ZFS}}, \mathrm{NRM}_{+-}\right)$and the corresponding viscous components subtracted $\left(\mathrm{VRM}_{\mathrm{ZFS}}, \mathrm{VRM}_{+-}\right)$.

thermally activated flips of spontaneous magnetizations (e.g. an enhancement of $\mathrm{VRM}_{+-}$magnitude) would imply that $\mathrm{NRM}_{+-}$magnitude should be lower than $\mathrm{NRM}_{\text {ZFS }}$, which is not the case (Fig. 2). Coercivity spectra obtained from AF demagnetization of natural samples with an acquired laboratory VRM (Fig. 3) show an increased importance of very soft carriers in older palaeosol units, thus causing a shift in coercivity spectra towards low fields. Since we did not carry out AF demagnetization before the VRM acquisition experiment, using only a 30-days ZFS, the shape and hardness of the coercivity spectra probably are influenced by a stable NRM component residing in the $<20 \mathrm{mT}$ coercivity region.

The experiments carried out on the second set of palaeosol samples were aimed at revealing any peculiarities in the process of VRM acquisition, which could explain the observed differences in the VRM amplitudes depicted in Fig. 2. In order to have the same initial state, we prepared this new set of samples, rather than using the already treated samples from set 1 . Figure 4 shows: i) VRM acquisition (circles, initial state: 3 days of ZFS of the samples with NRM signal); ii) acquisition of VRM in opposite $(-Z)$ direction with an initial state of (-VRM) remanence acquired after the first acquisition (diamonds); iii) the third cycle of VRM acquisition from an initial state (-VRM) after ii) (triangles). The curves are plotted without subtracting the initial $(t=0)$ signal. All the samples subjected to this procedure show linear with $\log (t)$ VRM acquisition for periods of 7 days $\left(10^{4}\right.$ $\mathrm{min}$.). The calculated from the linear regression line Sa values for different palaeosol horizons (Table 1) vary in the range $2.37-0.37 \times 10^{-9} \mathrm{Am}^{2} / \mathrm{kg}$ for the initial acquisition (Sa1) and $4.46-0.86 \times 10^{-9} \mathrm{Am}^{2} / \mathrm{kg}$ for the second acquisition (Sa2), having a maximum in $\mathrm{S}_{3}$ unit. The ratio $\mathrm{Sa}(2) / \mathrm{Sa}(1)$ of close to 2 reflects the fact that the second VRM acquisition started from an initial reversed magnetization (-VRM, e.d. 

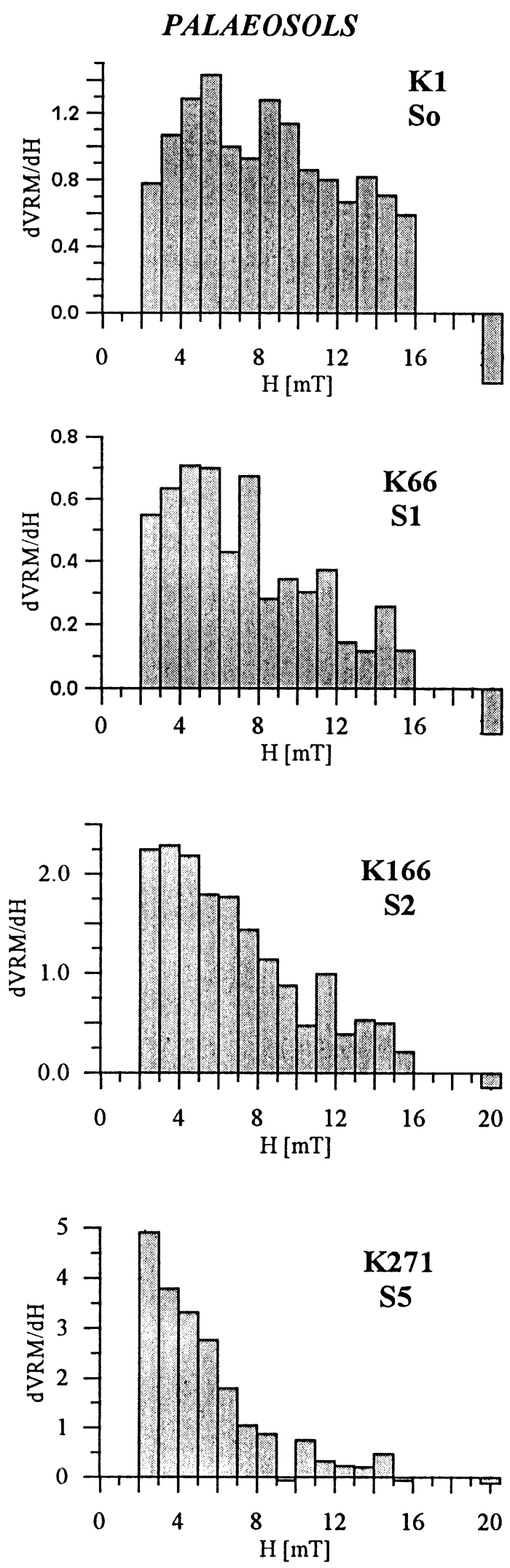

LOESSES
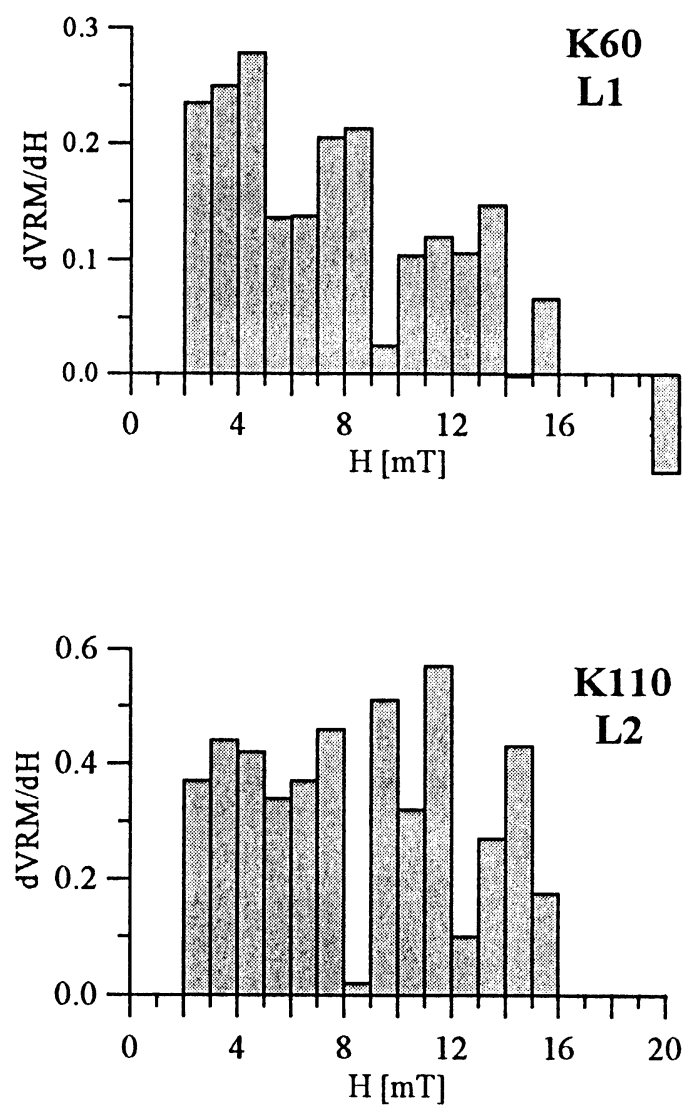

Fig. 3. Coercivity spectra for some representative samples from set 1 obtained through AF demagnetization of laboratory VRM. 
Table 1. Magnetic susceptibility, viscous acquisition coefficients $\mathrm{Sa}(1)$ and $\mathrm{Sa}(2)$ obtained from two different initial states (ZFS and - VRM) and their ratio for non-oriented palaeosol samples.

\begin{tabular}{cccccc}
\hline Unit & Sample No. & $\mathrm{Sa}(1)\left(10^{-9} \mathrm{Am}^{2} / \mathrm{kg}\right)$ & $\mathrm{Sa}(2)\left(10^{-9} \mathrm{Am}^{2} / \mathrm{kg}\right)$ & $\mathrm{Sa}(2) / \mathrm{Sa}(1)$ & $\chi\left(10^{-6} \mathrm{~m}^{3} / \mathrm{kg}\right)$ \\
\hline palaeosol & K 69 & 0.375 & 0.862 & 2.3 & 0.443 \\
S1 & K 75 & 0.774 & 1.613 & 2.09 & 0.702 \\
palaeosol & K 164 & 0.839 & 1.625 & 1.94 & 0.726 \\
S2 & K 169 & 0.961 & 1.959 & 2.04 & 0.882 \\
palaeosol & K 202 & 2.054 & 4.040 & 1.97 & 1.868 \\
S3 & K 204 & 2.372 & 4.455 & 2.04 & 1.636 \\
palaeosol & K 232 & 1.699 & 3.475 & & 1.255 \\
S4 & & & & 1.93 & 0.958 \\
palaeosol & K 262 & 1.426 & 2.752 & 2.2 & 1.131 \\
S5 & K 270 & 1.514 & 3.359 & 1.86 & 0.756 \\
palaeosol & K 293 & 0.947 & 1.759 & 2.18 & 1.111 \\
S6 & K 313 & 1.544 & 3.366 & 1.9 & 0.583 \\
\hline
\end{tabular}

"saturated" viscous moments in oposite direction), while the first VRM acquisition started from a state after ZFS (random orientations of individual unstable magnetizations). Several normalization parameters are used-susceptibility $(\chi)$, saturation magnetization (Js), and percent frequency dependent magnetic susceptibility $\left[\chi \mathrm{fd} \%=\left(\chi_{\mathrm{LF}}-\chi_{\mathrm{HF}} / \chi_{\mathrm{LF}}\right) * 100\right]$, obtained for companion samples. The each one reduces the role of concentration on $\mathrm{Sa}$ values in a different manner. Saturation magnetization and susceptibility, which sum the contribution of the total ferromagnetic content (not only of the viscous fraction), are appropriate normalization parameters when there are similar proportions of stable grains in the assembly (e.g. only palaeosols or loesses). The third parameter $-\chi_{\mathrm{fd}} \%$ is influenced only by the SP fraction close to the SP/SD boundary and thus would better represent only the varying contribution of viscous grains in different lithological horizons. The significant role of concentration in determination of Sa values is clearly indicated by the well expressed dependence between $\mathrm{Sa}$ and magnetic susceptibility (Fig. 5(a)). On the other hand, the observed linear dependence between the values of $\mathrm{Sa}$ and frequency dependent magnetic susceptibility $\left(\chi_{\mathrm{LF}}-\chi_{\mathrm{HF}}\right)($ Fig. $5(\mathrm{~b}))$ suggests the presence of continuous (uniform) distribution of relaxation times from $10^{-4} \mathrm{sec}$. (Worm, 1998) up to $10^{4} \mathrm{~min}$. The plot of Sa values normalized by Js ( $\left.\mathrm{Sa} / \mathrm{Js}_{\mathrm{s}}\right)$ as a function of the ratio $(\chi / \mathrm{Js})$ (Fig. 6(a)) shows also that the process of viscous acquisition (e.g. Sa) is influenced by the presence of SP grains, as far as $\chi / \mathrm{Js}$ is used as a measure of SP contribution (Dunlop, 1981; Banerjee, 1994). The shift up of the experimental points, corresponding to $\mathrm{Sa} 2$ values, reflects the fact that $\mathrm{Sa} 2 / \mathrm{Sa} 1 \sim 2$ due to the initial state $(-\mathrm{VRM})$ before subsequent acquisition in the opposite direction. Otherwise, no systematic difference is observed between Sa values obtained for set 2 (initial state ZFS) and set 3 (initial state: AF demagnetization at $100 \mathrm{mT}$ ) (Fig. 6(a)). The same linear relationship is obtained if we use $\chi_{\mathrm{fd}} \%$ as a normalizing parameter for Sa (Fig. 6(b)). Consequently, the main contribution to Js comes from the fine-grained viscous fraction.

The last set of experiments was carried out on artificially prepared samples from $0.2 \mathrm{~mm}$ mechanical fraction and were used to investigate the viscous acquisition in laboratory field and subsequent decay in zero field. Using artificial samples, we eliminate the effect of any initial remanence on viscous acquisition and stability of the viscous remanence itself. In contrast, in our previous experiments with samples from set 1 and set 2, we had to consider the possible influence of the stable component. For an acquisition period of 30 days, we obtained quite good linear VRM(log $t)$ curves (Fig. 7). The calculated Sa values, as well as their normalized equivalents (to susceptibility and Js) are listed in Table 2. In addition to palaeosol samples, which contain a significant SP fraction, we have included in this series samples from the parent loses in order to compare the palaeosol behaviour with nonenhanced (unweathered) material. It is likely that viscous acquisition is due mainly to the pedogenic (SP/SD) fraction, not to a possible contribution from MD grains of detrital magnetites (or TM), considering the significantly lower Sa values of loess samples. Figure 7 shows some typical examples of 30-days VRM acquisition and subsequent decay in zero field for a period of 4 to 5 hours. The most pronounced non-linear $\operatorname{VRM}(\log t)$ decay behaviour is typical for the first $10 \mathrm{~min}$. after the sample is inserted into the measuring coil. Particular two-stage decay behaviour is observed for sample K271 from the most enhanced with SP grains palaeosol horizon $\mathrm{S}_{5}$ (Jordanova and Petersen, 1999). Comparing the behaviour of acquisition and decay processes (Fig. 7), we have calculated the slopes of the linear parts from the VRM-decay curves starting from $t=1000 \mathrm{sec}$., in order to be closer to the time interval represented in the acquisition experiments. For comparison, Fig. 8 shows the VRM decay behaviour of one sample of known mineralogy $\left(\mathrm{Fe}_{3} \mathrm{O}_{4}\right)$ and grain size $(d=0.2$ $\mu \mathrm{m})$. Identification of magnetic mineralogy through thermal 

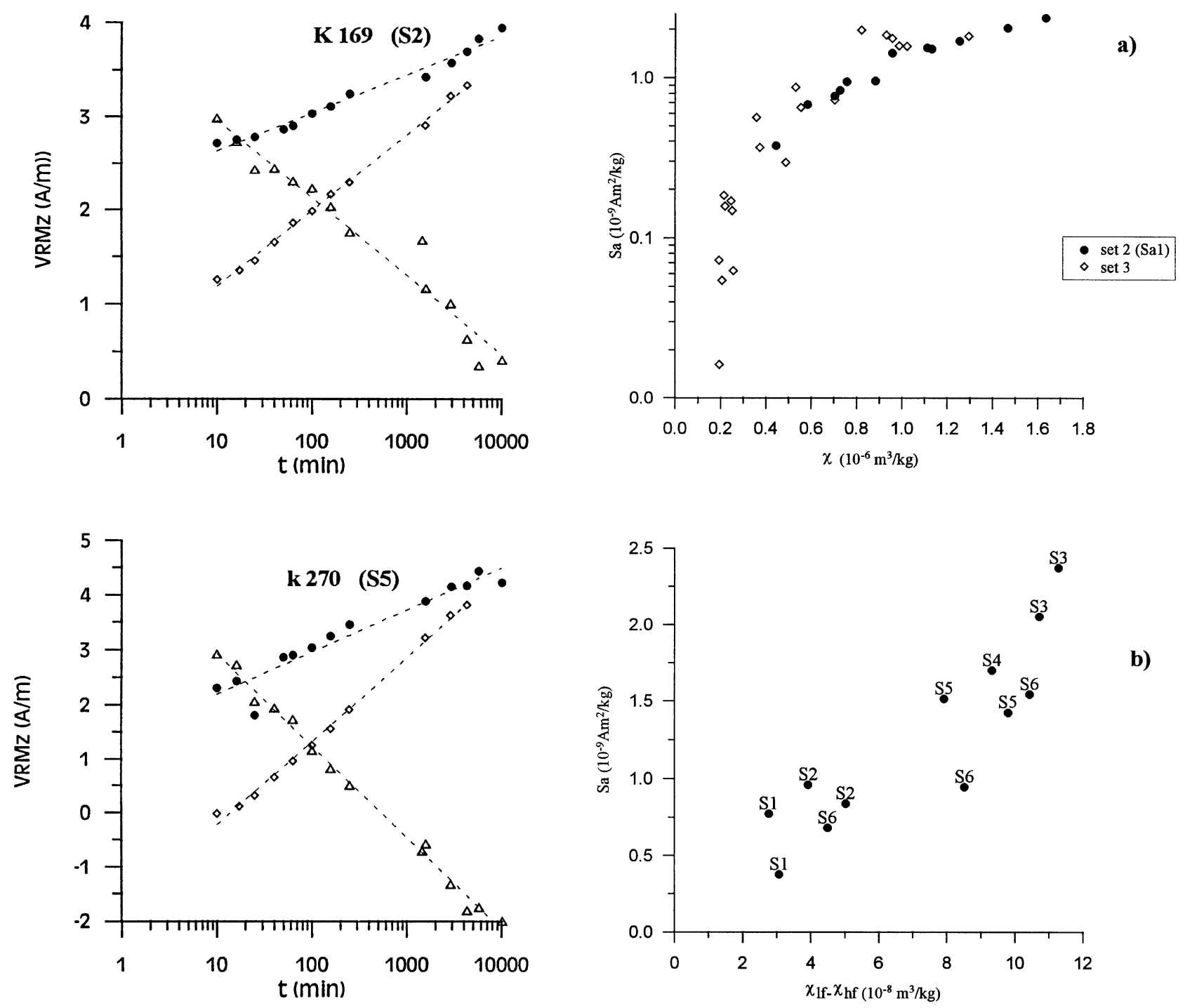

b)

Fig. 5. a) Dependence of Sa on susceptibility $(\chi)$. Set 2 (dots) and set 3 (open diamonds) b) correlation between frequency dependent magnetic susceptibility $\left(\chi_{\mathrm{LF}}-\chi_{\mathrm{HF}}\right)$ and viscous acquisition coefficients $\mathrm{Sa}(1)$.

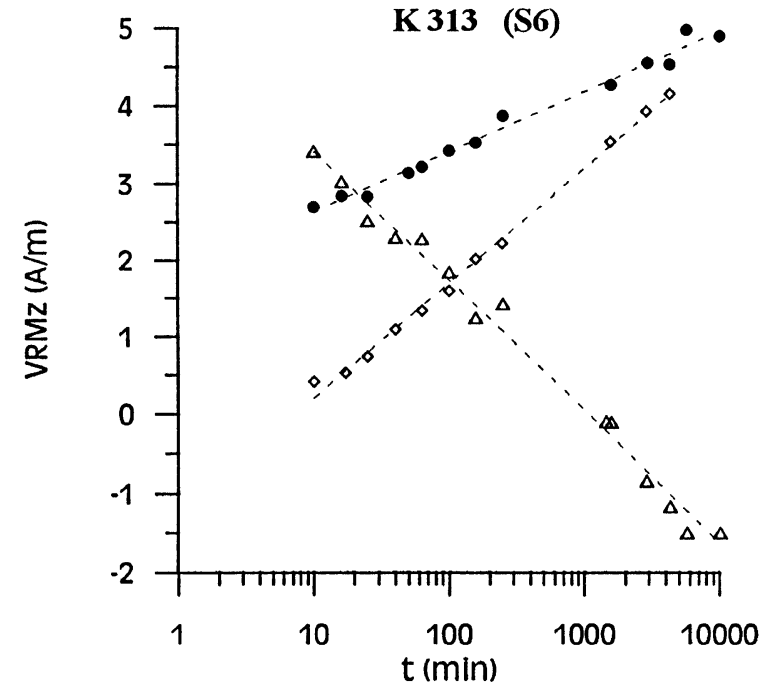

Fig. 4. Viscous acquisition of samples from set 2, starting from different initial states: full circles-VRM acquisition in $(+z)$ direction after 5-6 days of ZFS; open diamonds-re-acquisition in an opposite $(-z)$ direction; open triangles - subsequent acquisition in $(+z)$ direction. demagnetization of saturation remanence, acquired in a field of $2 \mathrm{~T}$, shows only a magnetite $\mathrm{Tb}$ of $580^{\circ} \mathrm{C}$ (Fig. 8(a)). A linear VRM $(\log t)$ decay curve is obtained also for this sample (Fig. 8(b)).

Experiments on AF demagnetization of viscous remanences, acquired during different times of exposure to the laboratory field, have also been carried out. The results are consistent with the classical understanding of thermally activated moments, according to which the coercivity, as well as the intensity of VRM, increases with increasing acquisition time (Fig. 9). The greater (proportionally) part of undemagnetized moment for the shortest acquisition time $(20 \mathrm{~min}$.) in fields higher than $8 \mathrm{mT}$ most probably reflects a higher noise-to-signal ratio for the weakest remanence signals.

\section{Discussion}

The simplest way of viscous magnetic cleaning, applied in many palaeomagnetic laboratories, is performed either by zero-field storage of samples for few weeks (Banerjee, 1981) or double equal-time exposure in two opposite positions in 
a)
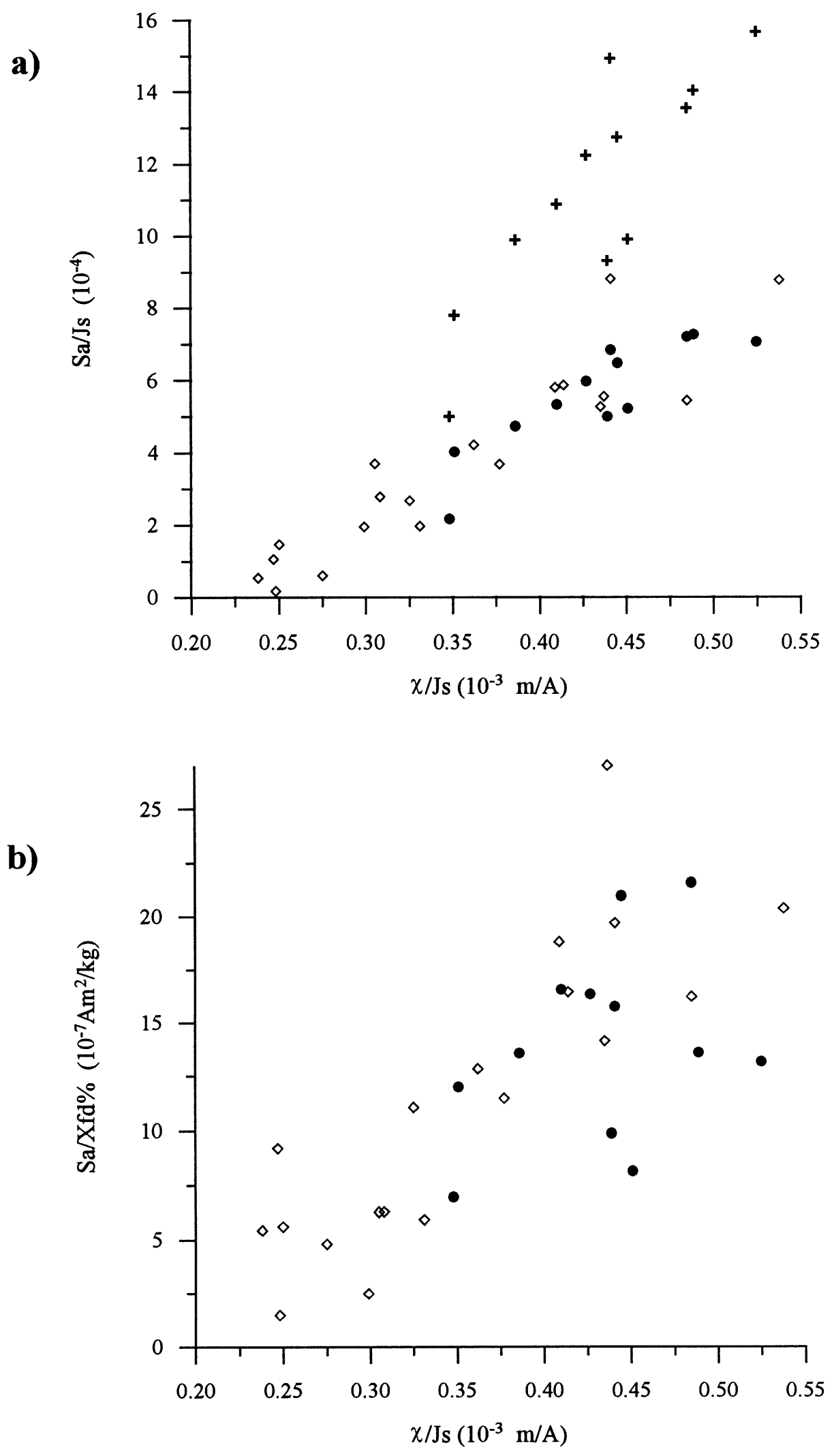

- set 2 (Sal)

$\diamond$ set 3

Fig. 6. Correlation between the normalized acquisition coefficients $\mathrm{Sa} / \mathrm{Js}$ (a) and $\mathrm{Sa} / \chi \mathrm{fd} \%$ (b) and the ratio $\chi / \mathrm{Js}$. 


\section{K1 (So)}
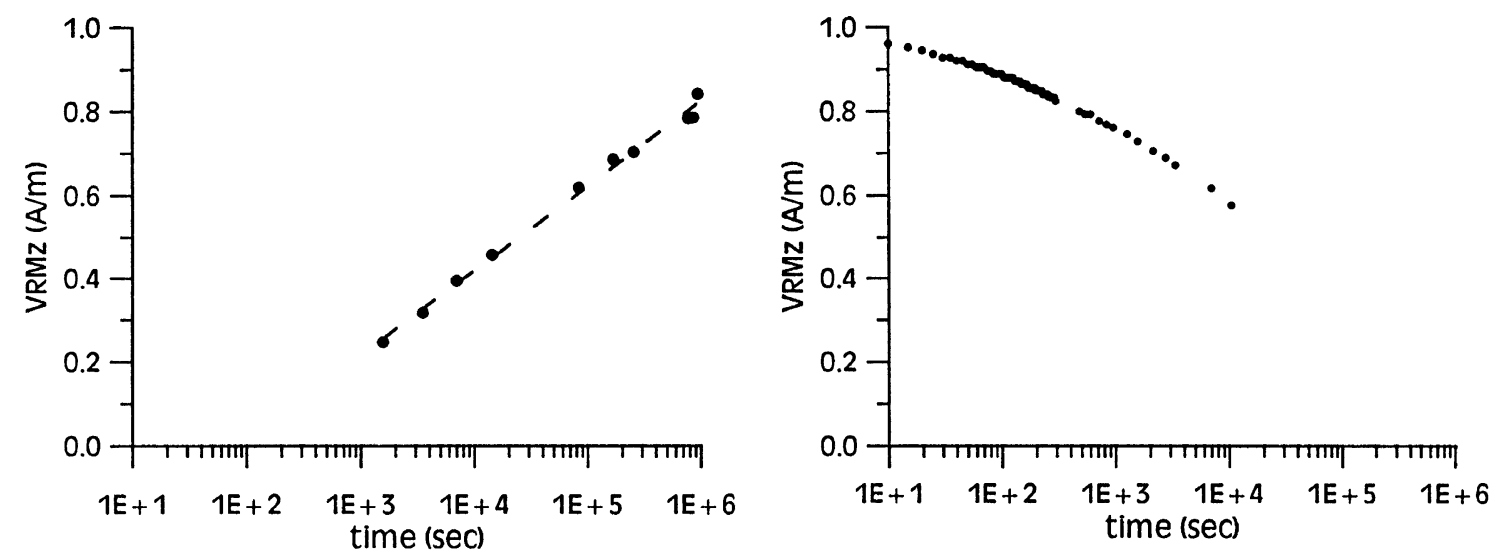

\section{K66 (S1)}
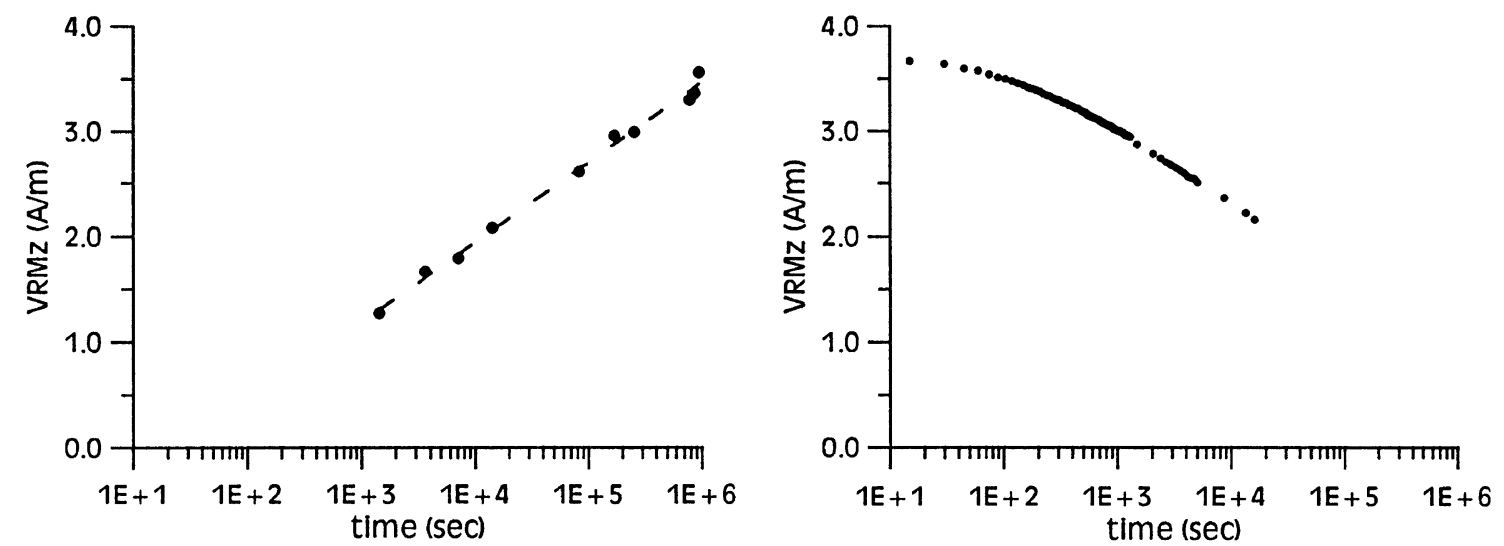

K271 (S5)
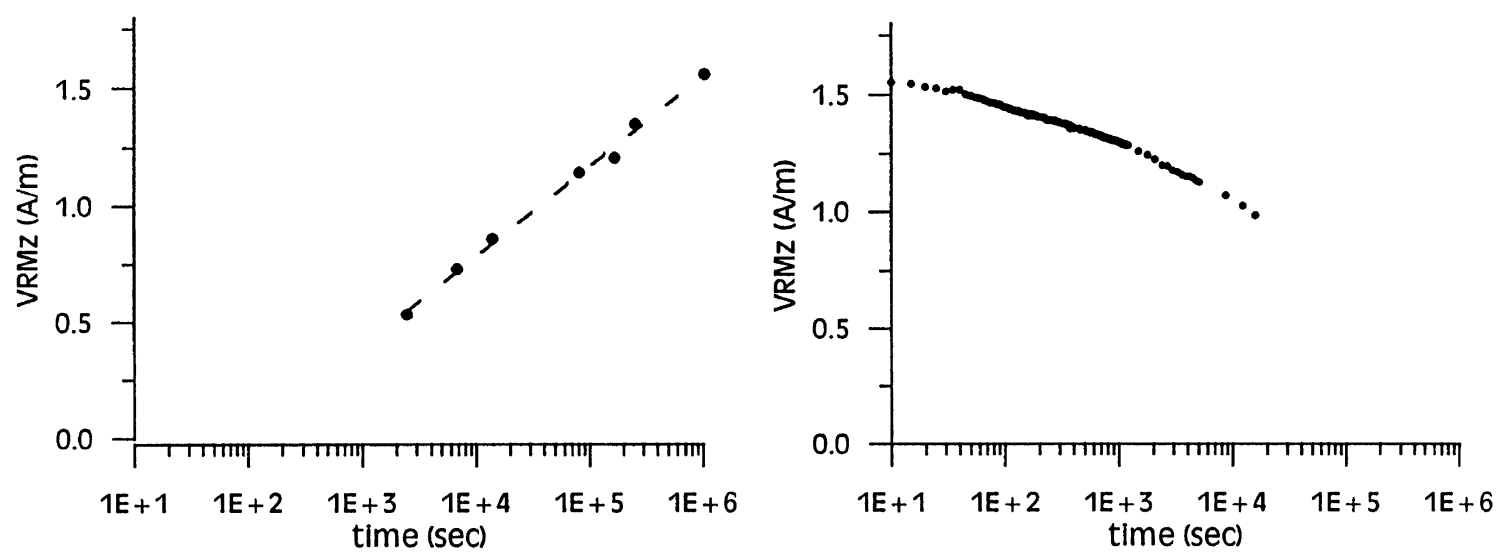

Fig. 7. Acquisition of laboratory VRM and its decay in zero field starting from an initial AF demagnetized state.

relation to the local magnetic meridian (Chramov, 1982). Tentatively, these methods should erase the soft viscous (parasitic) remanence, although the hardest part of VRM will remain unchanged. In our study we applied initially the first approach, leaving the samples from set 1 in $\mu$-metal boxes for a period of 30 days. It is obvious from Fig. 2 that the obtained variations of the viscous and stable remanences parallel susceptibility behaviour, suggesting that the concentration of the ferromagnetic carriers is the main controlling factor. It is worth mentioning, however, that viscous component as a 
Table 2. Magnetic susceptibility, coefficients of viscous acquisition Sa and their normalized values for the artificially prepared samples from set 3.

\begin{tabular}{|c|c|c|c|c|}
\hline Unit & Sample No. & $\mathrm{Sa}\left(10^{-9} \mathrm{Am}^{2} / \mathrm{kg}\right)$ & $\mathrm{Sa} / \mathrm{Js} \times 10^{-4}$ & $\chi\left(10^{-6} \mathrm{~m}^{3} / \mathrm{kg}\right)$ \\
\hline recent soil & K 1 & 0.728 & 3.695 & 0.702 \\
\hline \multicolumn{5}{|l|}{ S0 } \\
\hline \multirow[t]{3}{*}{ loess L1 } & K 27 & 0.147 & 1.064 & 0.251 \\
\hline & K 50 & 0.063 & 0.605 & 0.255 \\
\hline & K 60 & 0.054 & 0.545 & 0.205 \\
\hline palaeosol & K 66 & 0.365 & 2.676 & 0.371 \\
\hline $\mathrm{S} 1$ & K 70 & 0.655 & 3.691 & 0.553 \\
\hline \multirow[t]{3}{*}{ loess L2 } & K 80 & 0.296 & 2.793 & 0.486 \\
\hline & K 101 & 0.073 & 0.727 & 0.191 \\
\hline & K 110 & 0.016 & 0.174 & 0.194 \\
\hline palaeosol & K 157 & 1.581 & 5.806 & 0.987 \\
\hline S2 & K 166 & 0.874 & 4.223 & 0.529 \\
\hline loess L3 & K 189 & 0.157 & 1.456 & 0.219 \\
\hline palaeosol & K 205 & 1.819 & 5.439 & 1.294 \\
\hline S3 & K 210 & 1.571 & 5.267 & 1.022 \\
\hline loess L4 & K 220 & 0.169 & 1.957 & 0.245 \\
\hline palaeosol & K 231 & 1.761 & 5.867 & 0.958 \\
\hline \multicolumn{5}{|l|}{$\mathrm{S} 4$} \\
\hline palaeosol & K 271 & 1.854 & 8.772 & 0.931 \\
\hline \multicolumn{5}{|l|}{ S5 } \\
\hline loess L6 & K 278 & 0.567 & 5.557 & 0.357 \\
\hline palaeosol & K 311 & 1.990 & 8.812 & 0.821 \\
\hline \multicolumn{5}{|l|}{ S6 } \\
\hline loess L7 & K 325 & 0.184 & 1.974 & 0.213 \\
\hline
\end{tabular}

part of the total (initial) NRM signal increases progressively towards the older palaeosol horizons. The progressive increase in VRM for the older samples cannot be attributed to increased acquisition time (the Matuyama/Brunhes boundary found in the seventh loess L7 (Hus et al., 1997)), since increased acquisition time is seen to harden the VRM (Prevot, 1981). The next set of experiments reveal another peculiarity in laboratory acquisition of viscous remenence. Starting from an initial state of ZFS, we induce VRM for the same time period (30 days) expecting to obtain a similar viscous component as the erased one. In order to compare the stable remanences as well, we applied a second viscous cleaning by inverting the samples for 30 days. As a result, the calculated values of stable remanence that remained after this second cleaning coincided with that obtained after ZFS (Fig. 2). However, the viscous component acquired in the laboratory was always significantly stronger than that erased during ZFS (Fig. 2). The difference depicted in Fig. 2 should be considered as an underestimation, as far as we have plotted the total $\mathrm{VRM}_{\text {ZFS }}$ (having in mind that the samples are non-oriented), but we have only used the VRM acquired along the $(+Z)$ direction in order to minimize some effects of changes in horizontal component of the laboratory magnetic field. As a result, we have the following situation:

1) equal amounts of stable remanences, remained after ZFS and double equal-time exposure to the laboratory field, and

2) significantly higher $\mathrm{VRM}_{+-z}$ in comparison with $V_{R M}$ ZF in spite of the suppressing effect of ZFS on subsequent viscous acquisition (Tivey and Johnson, 1984; Moskowitz and Banerjee, 1981). The applied procedure of ZFS for a period of 30 days will not destroy all the laboratory acquired VRM since the non-oriented pieces of the material have been stored before the experiments for about 6-7 months in the laboratory. In spite of the random direction of labVRM component thus acquired, the intensity of $\mathrm{VRM}_{\mathrm{ZFS}}$ will not depend on it, as far as the total vector is taken into consideration. Contrary, in calculation of $\mathrm{VRM}_{+-z}$ we took only the $z$-component.

Coercivity spectra, obtained through AF demagnetization of laboratory VRM, shown in Fig. 3, point to a progressively increasing softness of the remanence carriers in older 


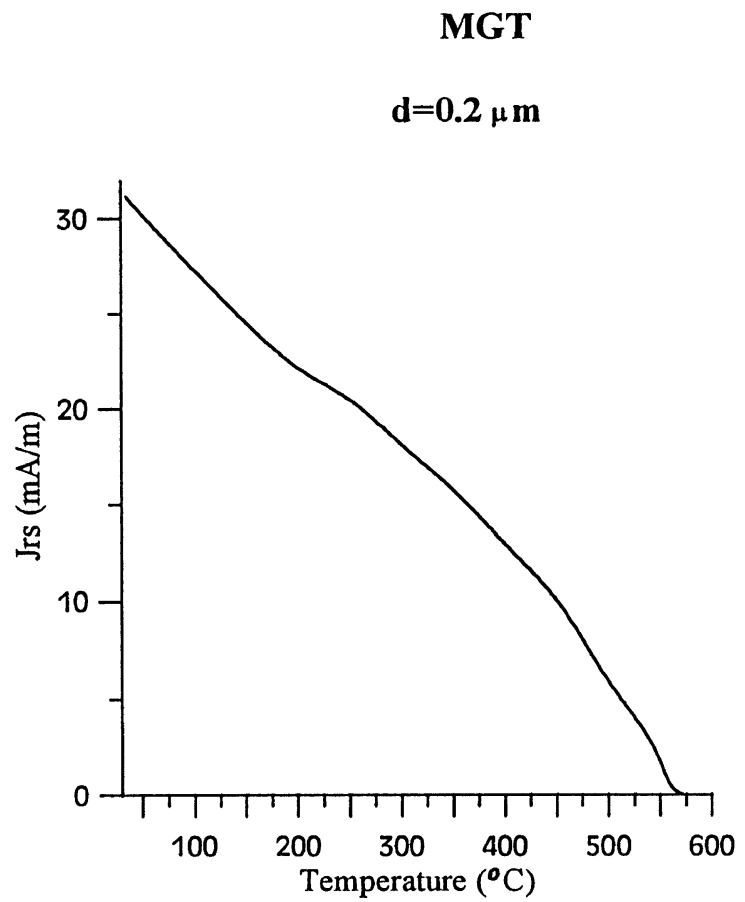

a)
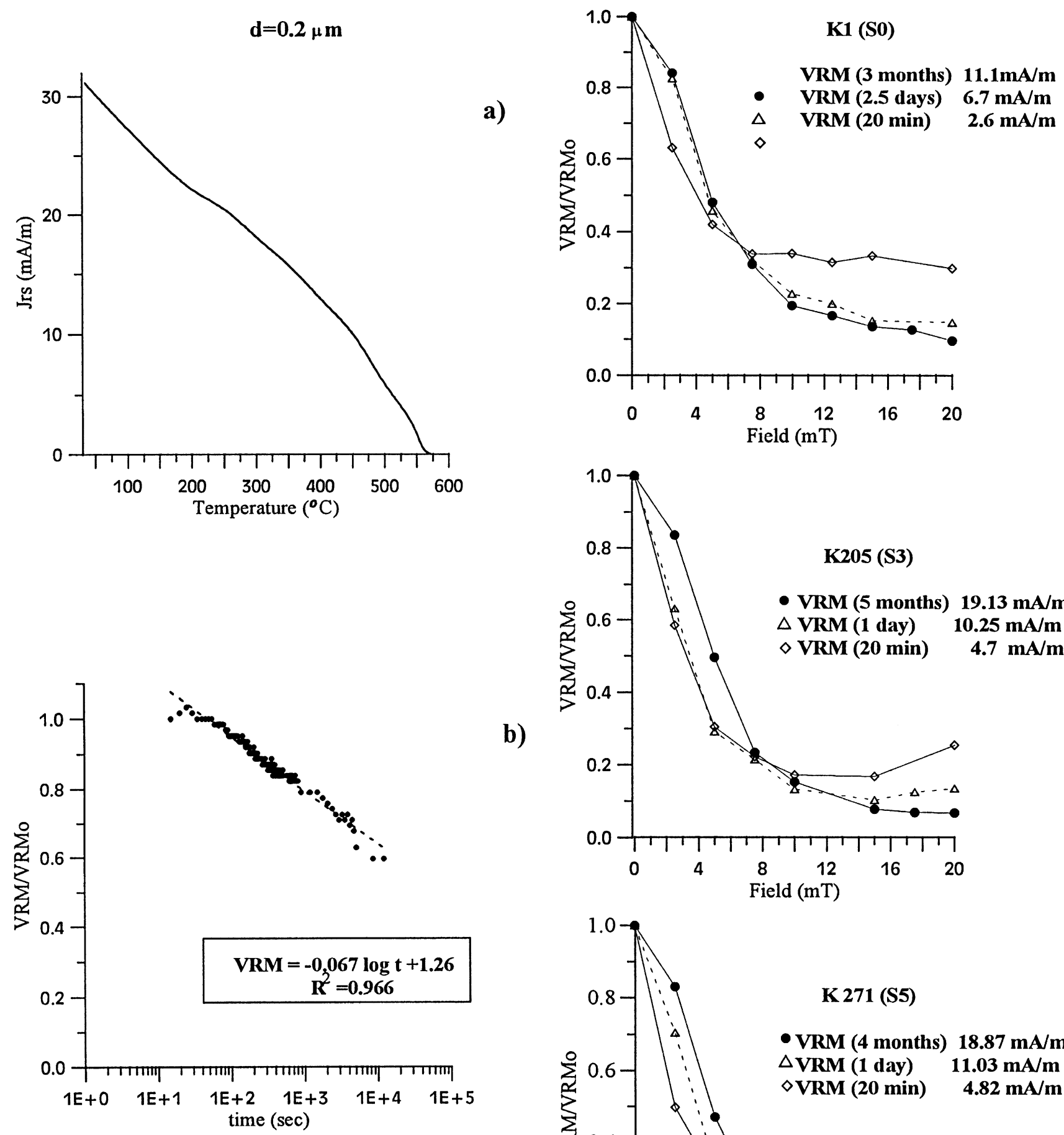

b)
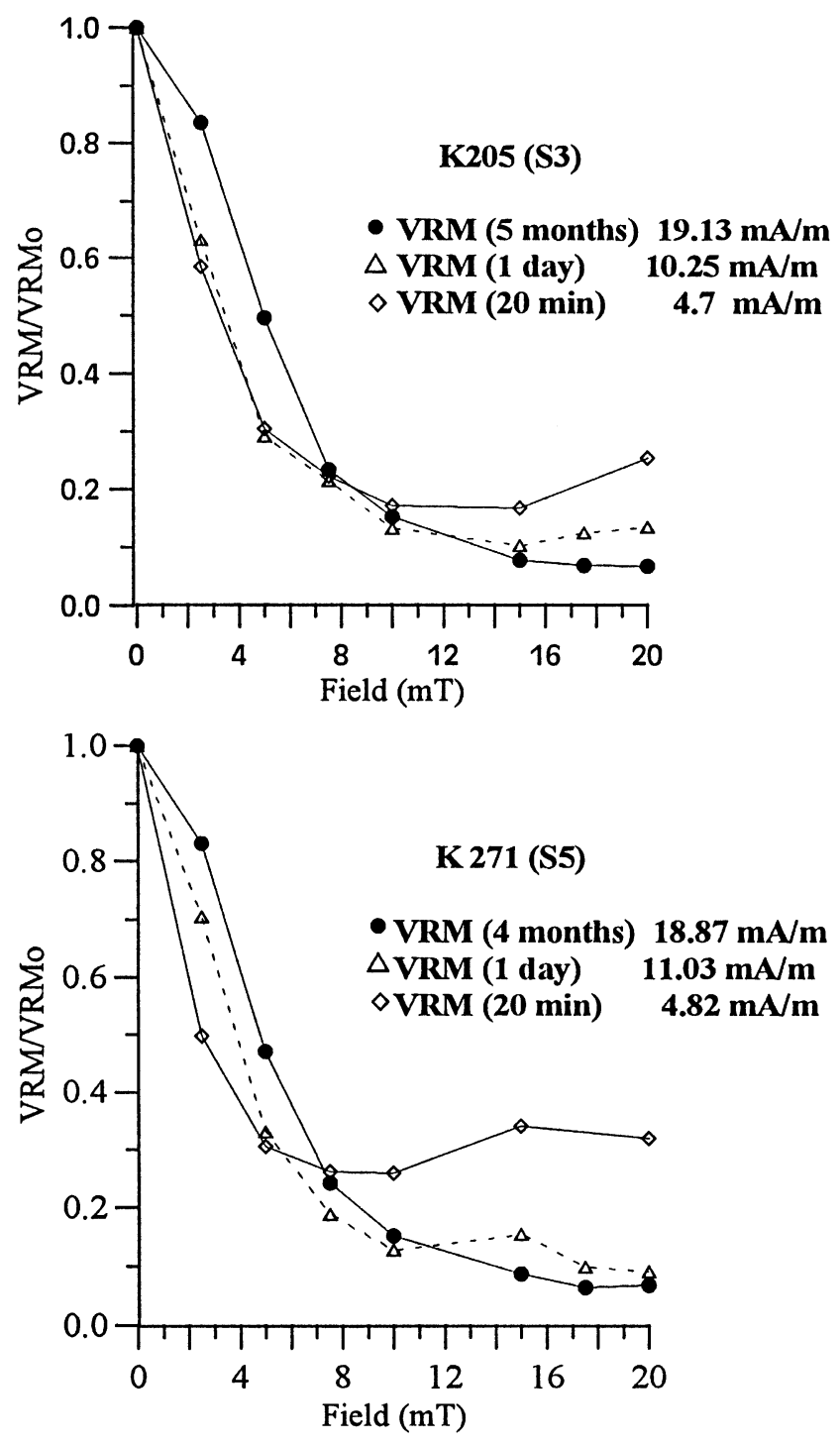

palaeosols, although there should be some influence of the underlying stable remanence component, which is stronger for the upper palaeosols and thus leads to the observed wider spectra. This ambiguity is explained by the AF demagnetizations of laboratory imparted VRMs in artificial samples with an initial AF demagnetized state (Fig. 9). Obviously, the main part of a 3-5 months VRM is demagnetized at about $8-10 \mathrm{mT}$, suggesting that higher fields already demagnetize

Fig. 9. AF demagnetization curves for VRMs, acquired for different times of exposure. All the curves are normalized to the corresponding initial VRM values. 
a part of the "stable" remanence in Fig. 3.

Magnetic mineralogy of the studied samples is not discussed in this paper, as far as the results from the thermomagnetic analyses and low-temperature demagnetization are presented in Jordanova and Petersen (1999). It was shown that the main ferromagnetic mineral is magnetite, oxidized to a different degree in different palaeosol units. Recent soil So shows mainly a maghemite presence, while older palaeosols are thought to contain partially oxidized magnetite grains of predominantly SP/SD sizes, probably with $\mathrm{Fe}_{3} \mathrm{O}_{4}$-core $/ \gamma \mathrm{Fe}_{2} \mathrm{O}_{3}$-shell structure. On the basis of this ferromagnetic mineralogy of the samples, we would expect some differences in the viscosity parameters among different palaeosol horizons, having in mind that the low temperature (LT) oxidation can influence magnetic viscosity (Ozdemir and Banerjee, 1981; Moskowitz and Banerjee, 1981). Experimental procedures designed for samples of set 2 were intended to reveal whether this LT oxidation will influence the shape of the VRM acquisition curves.

Some representative examples of subsequent VRM acquisition, are shown in Fig. 4. The observed linear VRM( $\log t)$ dependence for all three viscous acquisitions points to a uniform distribution of relaxation times (Dunlop and Ozdemir, 1997) up to 7 days for all the samples studied. The theoretically predicted $\mathrm{Sa}(2) / \mathrm{Sa}(1)=2$ value (Dunlop and Hale, 1977), agrees well with most of our samples (Table 1), which fall in the range from 1.86 to 2.3. Probably one of the reasons for the deviations in $\mathrm{Sa}(2) / \mathrm{Sa}(1)$ ratio from 2 is the quality of the regression from which corresponding Sa coefficients have been determined.

As it was shown by the previous considerations, LT oxidation (undoubtedly present in our samples) did not affect the time-dependence of VRM. However, if LT oxidation has proceeded to a higher degree, the development of surface cracks will result in subdivision of the original grains and appearance of new SP regions (Dunlop and Ozdemir, 1997). This will be reflected by quantitative changes in the measured parameters which show grain-size dependence. Figure 6(a) shows the existence of a correlation between normalized $\mathrm{Sa} / \mathrm{Js}$ values and the ratio $\chi / \mathrm{Js}_{\mathrm{s}}$, the latter being a sensitive indicator for the presence of SP grains. A schematic representation of the gradual increase in $\mathrm{Sa} / \mathrm{J}$ s towards older palaeosols is given in Fig. 10 and seen in Table 2. It supports the hypothesis about the role of LT oxidation mentioned above. In addition to the two acquisition coefficients ( $\mathrm{Sa}(1)$ and $\mathrm{Sa}(2)$ ) obtained for the samples from set 2, we have plotted also the Sa values for samples from set 3, which are initially AF demagnetized before VRM acquisition. The presence of a single trend of linear $\mathrm{Sa} / \mathrm{Js}_{\mathrm{s}}=\mathrm{f}\left(\chi / \mathrm{Js}_{\mathrm{s}}\right)$ dependence for set 3 and Sal of set 2, reveals that the difference in the initial states (AF demagnetization and ZFS correspondingly) do not influence the ability of viscous acquisition in our case, in contrast to the results presented by Lowrie and Kent (1978).

A comparison between acquisition and decay of a laboratory VRM is shown in Fig. 7. In the decay experiments, we were able to measure viscous changes from an earlier stage, about $10 \mathrm{sec}$. after shielding of the laboratory magnetic field. Obviously a non-linear VRM vs. $\log t$ behaviour is typical for the first $1000 \mathrm{sec}$. Starting from this point, VRM decay

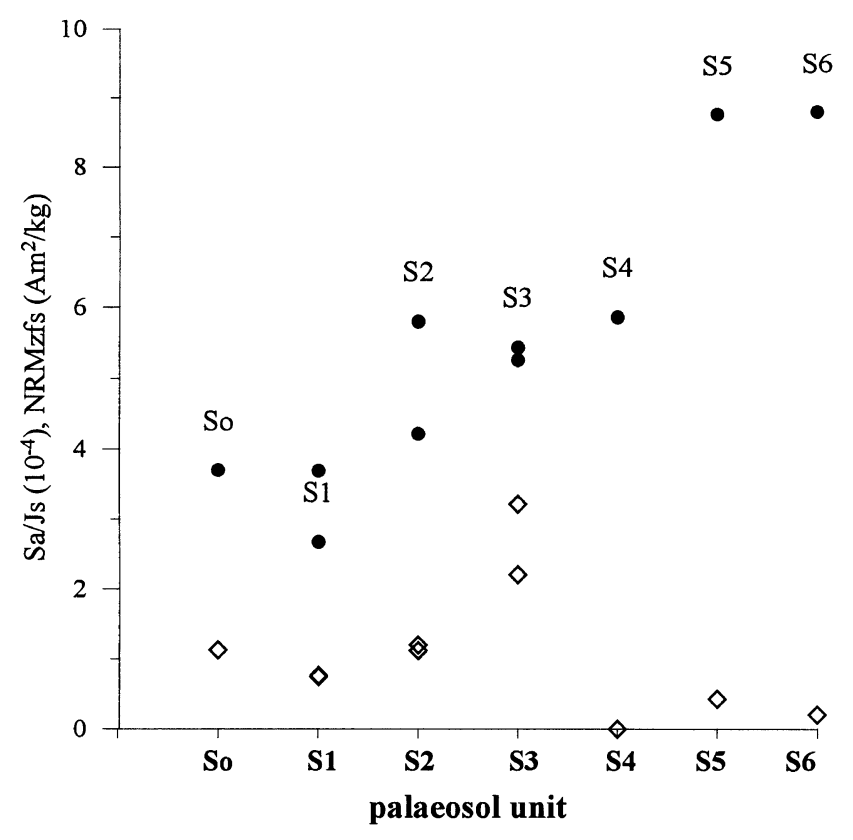

Fig. 10. Schematic representation of the increase of viscosity (Sa/Js) (circles) and the decrease of the magnitude of the stable remanence NRM (open diamonds) towards older palaeosol horizons.

curves can be fit successfully with linear extrapolation and coefficient of viscous decay $(\mathrm{Sd})$ can be obtained. For the samples studied, there is no consistent relationship between the calculated values of Sa and Sd. However, the amount of viscous remanence for each sample, that decays in $10^{4} \mathrm{sec}$, is always about 30\% lower than the VRM acquired for the same time period. This observation is consistent with the considerations of Dunlop (1973) and Worm et al. (1988), where it is shown that for an assembly of SD particles after equally long acquisition and decay times, $37 \%$ of the acquired magnetization still survives during VRM decay in zero field. However, this is not observed for sample K1 from the recent soil So, where VRM decay removes almost all of the VRM acquired.

A particularly interesting problem in our study involves the existence and properties of chemico-viscous remanence in palaeosol samples. CVRM is shown to appear during the process of low-temperature oxidation of an initial SD magnetite phase (Ozdemir and Dunlop, 1989; Gapeev et al., 1991) and increases logarithmically with time. It can be considered as a sum of unstable (with respect to time) VRM and stable ("true") CRM. We have to take into account that in our study we are dealing with such a CVRM during the initial ZFS of samples from set 1 . Compared to the uppermost soil horizons, older palaeosols (S5, S6) had relatively lower values of the stable signal $\left(\mathrm{NRM}_{\mathrm{ZFS}}\right)$ remaining after 30 days of ZFS, accompanied by well expressed increase in viscosity for S4, S5, and S6 (Fig. 10). This indicates that low-temperature oxidation caused the appearance of an extra-amount of SP viscous grains in expense of the stable NRM carriers probably through their subdivision by surface cracks. The obtained low values of the Median Destructive Fields (MDFs between 4 and $8 \mathrm{mT}$ ) suggest that most probably it is not strongly coupled with the true CRM (or stable NRM) components and is aligned with the applied field. Another supposition 
is that the type of pedogenesis also plays a role for the relative proportion of stable (primary) and secondary viscous pedogenic grains. The importance of moisture regime on the production of SP magnetites (Thompson and Maher, 1995) suggests that during more humid conditions higher amount of fine grained magnetic fraction (the latter being the source of $\mathrm{NRM}_{\text {ZFS }}$ ) is produced. Palaeopedological information available (Minkov, 1968) points to the presence of significantly more humid climates existing during the formation of older palaeosols (S4 to S6).

Concerning the observed non-linear VRM vs. $\log t$ behaviour of the decay curves (Fig. 7), we cannot ascribe it to the presence of significant MD fraction, as suggested by Dunlop (1981), because of the obtained linear VRM vs. $\log t$ dependence for the additionally studied sample of natural $\mathrm{Fe}_{3} \mathrm{O}_{4}$ crystals with pseudo single domain (PSD) $(d=0.2$ $\mu \mathrm{m}$ ) sizes (Fig. 8). A more likely supposition is that the obtained peculiarities are related to the presence of different amounts of ultra fine SP grains, having in mind the obtained correlation between $\mathrm{Sa} / \mathrm{JS}_{\mathrm{S}}$ and $\chi / \mathrm{JS}_{\mathrm{S}}$ values (Fig. 6).

The implication of the results from our study to the palaeomagnetism of loess/soil sediments concerns our observation that this material acquires a very strong viscous component, which can be build up in a short time period during laboratory storage of the samples. Erasing simply the short-time VRM (ZFS of 30 days) of the non-oriented samples from set 1 leads to a 2.5 times increase of the standard deviation of the population of measured inclinations $\left(\mathrm{STDEV}=\operatorname{SQRT}\left\{\left(n \Sigma x^{2}-(\Sigma x)^{2} / n^{2}\right\}\right)\right.$ after storage. The importance of the soft viscous remanence in the NRM signal increases significantly towards older palaeosol horizons, suggesting that LT oxidation has proceeded to a degree which can cause destruction of the primary remanence. The latter can be easily seen from the plot used in Fig. 10.

\section{Conclusions}

1. Magnetic viscosity in palaeosol samples from a section in NE Bulgaria is due to the presence of a fine grained SP/SD authigenic fraction.

2. Viscous acquisition is most enhanced in palaeosol units for which core/shell structure of partially oxidized grains is supposed to exist on the basis of the results from rock magnetic measurements.

3. Progressive increase in the degree of low temperature oxidation and/or an increase of climate humidity towards older palaeosols is revealed.

Acknowledgments. We are gratefull to the two referees (Koji Fukuma and Tim Rolph) for their constructive comments, which helped very much for the improvement of the manuscript.

\section{References}

Banerjee, S., Experimental methods of rock magnetism and paleomagnetism, Advances in Geophysics, 23, 25-95, 1981.

Banerjee, S. K., Contributions of fine-particle magnetism to reading the global paleoclimate record (invited), J. Appl. Phys., 75(10), 5925-5930, 1994.
Chramov, A., Palaeomagnetism, pp. 90-92, Nedra, Leningrad, 1982 (in Russian).

Dunlop, D., Theory of the magnetic viscosity of lunar and terrestrial rocks, Rev. Geophys., 11, 855-901, 1973.

Dunlop, D., The rock magnetism of fine particles, Phys. Earth Planet. Inter. 26, 1-26, 1981.

Dunlop, D., Viscous magnetization of 0.04-100 $\mu$ m magnetites, Geophys. J. R. astr. Soc., 74, 667-687, 1983.

Dunlop, D. and C. Hale, Simulation of long-term changes in the magnetic signal of the oceanic crust, Canadian Journal of Earth Sciences, 14, 716-744, 1977.

Dunlop, D. and O. Ozdemir, Rock Magnetism. Fundamentals and Frontiers, pp. 262-264, 272 pp., Cambridge Studies in Magnetism, Cambridge University Press, 1997.

Fine, P., K. Verosub, and M. Singer, Pedogenic and lithogenic contributions to the magnetic susceptibility record of the Chinese loess/palaeosol sequence, Geophys. J. Int., 122, 97-107, 1995.

Gapeev, A., S. Gribov, D. Dunlop, O. Ozdemir, and V. Scherbakova, A direct comparison of the properties of CRM and VRM in the low-temperature oxidation of magnetite, Geophys. J. Int., 105, 407-418, 1991.

Heller, F. and M. Evans, Loess Magnetism, Rev. Geophys., 33(2), 211-240, 1995.

Hunt, C. P., M. Singer, G. Kletetschka, J. Tenpas, C. Hunt, S. Banerjee, J. Han, P. A. Solheid, E. Oches, W. Sun, and T. Liu, Rock-magnetic proxies of climate changes in the loess-palaeosol sequences of the western Loess Plateau of China, Geophys. J. Int., 123, 232-244, 1995.

Hus, J. and R. Geeraerts, Palaeomagnetic and rock magnetic investigation of Late Pleistocene loess deposits in Belgium, Phys. Earth Planet. Inter, 44, 21-40, 1986.

Hus, J., R. Geeraerts, D. Jordanova, and J. Evlogiev, Magnetostratigraphy of loess-palaeosol sequence in Viatovo, near Russe (Bulgaria), IAGA 1997 Abstract Book, p. 65, 1997.

Jordanova, D. and N. Petersen, Palaeoclimatic record from a loess-soil profile in north-eastern Bulgaria-I. Rock-magnetic properties, Geophys. J. Int., 138(2), 520-532, 1999.

Lowrie, W. and D. Kent, Characteristics of VRM in oceanic basalts, $J$. Geophys., 44, 297-315, 1978.

Minkov, M., Loess in North Bulgaria, 202 pp., Bulg. Acad. Sci., Sofia, 1968 (in Bulgarian).

Moskowitz, B. and S. Banerjee, A comparison of the magnetic properties of synthetic titanomaghemites and some oceanic basalts, J. Geophys. Res., 86(B12), 11869-11882, 1981.

O'Reilly, W., Rock and Mineral Magnetism, pp. 120-122, Blackie, Glasgow and London, 1984.

Ozdemir, O. and S. Banerjee, An experimental study of magnetic viscosity in synthetic monodomain titanomaghemites: implications for the magnetization of the ocean crust, J. Geophys. Res., 86(B12), 11864-11868, 1981.

Ozdemir, O. and D. Dunlop, Chemico-viscous remanent magnetization in the $\mathrm{Fe}_{3} \mathrm{O}_{4}-\gamma \mathrm{Fe}_{2} \mathrm{O}_{3}$ system, Science, 243, 1043-1047, 1989.

Prevot, M., Some aspects of magnetic viscosity in subaerial and submarine volcanic rocks, Geophys. J. R. astr. Soc., 66, 169-192, 1981.

Thompson, R. and B. A. Maher, Age models, sediment fluxes and palaeoclimatic reconstructions for the Chinese loess and palaeosol sequences, Geophys. J. Int., 123, 611-622, 1995.

Tivey, M. and P. Johnson, The characterization of viscous remanent magnetization in large and small magnetite particles, J. Geophys. Res., 89(B1), 543-552, 1984.

Worm, H.-U., On the superparamagnetic-stable single domain transition for magnetite, and frequency dependence of susceptibility, Geophys. J. Int., 133, 201-206, 1998.

Worm, H. U., M. Jackson, P. Kelso, and S. Banerjee, Thermal demagnetization of partial thermoremanent magnetization, J. Geophys. Res., 93(B10), 12196-12204, 1988.

D. Jordanova (e-mail: vanedi@server.geophys.bas.bg), G. Yancheva, and V. Gigov 